\title{
The Complexity of Economic Policy: \\ I. Restricted local optima in tax policy design
}

\author{
Gilles Saint-Paul \\ GREMAQ-LEERNA-IDEI, \\ Université des Sciences Sociales de Toulouse
}

March 29, 2002 


\begin{abstract}
Economists traditionally tackle normative problems by computing optimal policy, i.e. the one that maximizes a social welfare function. In practice, however, a succession of marginal changes to a limited number of policy instruments are implemented, until no further improvement is feasible. I call such an outcome a "restricted local optimum". I consider the outcome of such a tatonment process for a government which wants to optimally set taxes given a tax code with a fixed number of brackets. I show that there is history dependence, in that several local optima may be reached, and which one is reached depends on initial conditions. History dependence is stronger (i.e. there are more local optima), the more complex the design of economic policy, i.e. the greater the number of tax brackets. It is also typically stronger, the greater the interaction of policy instruments with one another-which in my model is equivalent to agents having a more elastic labor supply behavior. Finally, for a given economy and a given tax code, I define the latter's average performance as the average value of the social welfare function across all the local optima. One finds that it eventually sharply falls with the number of brackets, so that the best performing tax code typically involves no more than three brackets.
\end{abstract}

JEL: H2, H21, J22

Keywords: Complexity, Optimal taxation, bouded rationality, learning, multiple equilibria, path dependence 


\section{Introduction}

Economists traditionally tackle normative problems by computing optimal policy, i.e. the one that maximizes a social welfare function. In practice, however, the actual conduct of economic policy rarely follows these guidelines. The whole set of government interventions-taxes, subsidies, and regulationsis characterized by a large set of parameters and, even if one were given an appropriate social welfare function, it is impossible to compute their optimal values. Rather, reform takes place as follows. There is status quo defined by a set of policy parameters inherited from the past, and there is a social demand to alter this status quo. Government respond to this status quo by altering a small subset of parameters, often marginally, i.e. by a small amount. The status quo gradually evolves as a result of this trial-and-error process. We expect the process to stop when no such marginal improvements are feasible.

Thus, rather than a first-best optimal policy being selected, the economy converges to a local optimum in a restricted sense, i.e. in the sense that the objective function cannot be improved by a small change to a small subset of parameters.

This raises a number of questions which do not arise in traditional optimal policy analysis. For example:

- To what extent is the resulting policy history-dependent? In a first best world, the optimum will be selected regardless of initial conditions. Under my trial and error assumption, if there are several restricted local optima, which one will eventually be selected will depend on initial conditions. The greater the number of local optima, the more arbitrary and history-dependent the policy outcome.

- How large are the losses that history-dependence may cause? In other words, do all local optima yield comparable values for the objective function, or are some of them clearly worse that others? 
- Should economic policy be simple or complex, that is, how many instruments should we use? In a first best world, an increase in the number of instruments is always beneficial, because it increases the set of policies that can be pursued (simple policies can always be replicated when more instruments are introduced). This is not necessarily true, however, in a trial-and-error world. Having more instruments could in principle increase the number of restricted local optima in such a way that some of them are worse than local optima under simpler policies. In other words, if complexity increases the number of local optima, it may make policy more history-dependent. This increases the likelihood of being remote from the best policy, i.e. makes large beneficial policy changes less likely to occur. In such a situation, one may make a case for simpler policies.

I analyze these issues in the context of a model of optimal taxation. The underlying structure of preferences and technology is standard. Agents differ in their ability as well as in their disutility of working. Income is taxed and the tax code is piece-wise linear, being characterized by $n$ tax brackets. Tax proceeds are used to finance a public good. The social welfare function depends on the level of the public good as well as on a composite index of income which reflects a concern about inequality. The only tax policy changes that are allowed are those which move one critical point in the tax schedule up or down by a small fixed amount. I call this a "feasible reform". A restricted local optimum (RLO) is reached when no feasible reform improves social welfare. I compute these local optima by starting from randomly selected tax codes, and then sequentially implementing feasible reforms, until an RLO is reached.

Our numerical results suggest that the number of RLOs goes up with the complexity of the tax system, as captured by the number of brackets. It also typically goes up when the curvature of the disutility of labor goes up, i.e. when labor supply is more reactive to changes in marginal tax rates. Another striking implication is that an increase in complexity beyond a cer- 
tain level is harmful to the expected performance of the tax system. That is, while greater complexity allows to better 'fine-tune' the best optimum, thus improving the corresponding value of the social welfare function, it also introduces new RLOs whose performance can be quite poor. I define the performance of the tax system as the average social level across RLOs, when each RLOs is weighted by the frequency with which is was reached across searches. When the number of tax brackets goes up beyond some point, performance deteriorates, implying that the welfare loss from reaching suboptimal equilibria more often outweigh the welfare gains from improving the value of the best optimum.

Our results are similar to those found by Kauffman (1985) in his so-called $N K$ model. This is an abstract statistical model which the author uses to analyze the evolution of complex biological objects such as proteins or DNA. A somewhat similar approach has been applied by Durlauf (1993) and Brock and Durlauf (2001) to economic problems such as ghetto formation and multiple long-run growth paths. The essence of these results is that path-dependence is more likely, the greater the complexity of the system and the greater the degree of interaction among units. Specifically, Kauffman considers $N$ units who can be in one of two boolean states ( 0 or 1 ), and whose individual contribution to the overall fitness of the system depends on the state of $K$ other units. By running numerous numerical simulations over randomly defined such systems characterized by $K$ and $N$, Kauffman shows that the "fitness landscape", i.e. the way the objective function varies over different states of the system, is more "rugged", i.e. has more local optima, the greater $K$ and $N$.

In my model, the equivalent of a unit is a given tax bracket, and the interaction among tax brackets is larger, the smaller the curvature of the disutility of effort. If this curvature is very strong, then labor supply is very inelastic to marginal income taxes, and so is income. The contribution of a given tax bracket to overall inequality and tax receipts is then basically independent of the level of other tax brackets, since workers have little opportunity to change 
tax brackets by changing their effort level. By contrast, if labor supply is very elastic, a change in a given tax bracket can induce a strong reallocation of workers among tax brackets. Thus interaction among tax brackets is stronger, the more elastic is labor supply. Finally, the equivalent of the biologist's fitness function is the social welfare function.

The similarity of our results with those of Kauffman are suggestive, but the present model is not a special case of his $N K$ model. While Kauffman considers any arbitrary, randomly generated, function defining the contribution of each unit to total fitness, in my model total fitness is the result of the underlying economic behavior of optimizing economic agents.

\section{The economy}

The artificial economies I consider have a standard structure. There are $q$ agents, who have access to a constant returns to scale technology producing a single homogeneous good using labor. Agents differ by their ability and their disutility of effort. Utility is given by

$$
U(c, e)=c-b e^{\eta} / \eta
$$

where $c$ is consumption, $e$ is effort, $b$ is the individual specific parameter capturing differences in the disutility of labor, and $\eta$ is a parameter capturing the curvature of this disutility. The greater $\eta$, the more inelastic is labor supply. As $\eta$ goes to infinity, labor supply becomes totally inelastic as each agent supplies one unit of effort.

The amount of good produced by an agent with ability $a$ is

$$
y=a e
$$

where $e$ is the agent's effort level. For simplicity, I assume that effort cannot exceed $1 / a$, so that total income is between 0 and 1 .

The tax code is a function relating net income $z$ to gross income $y$ :

$$
z=z(y)
$$


I assume that the tax code is continuous and piece wise linear over $n$ intervals, called 'brackets', whose $n+1$ kink points are equidistant; I restrict net income to be also between 0 and 1. A typical tax code is illustrated in Figure 1. Decreasing portions, i.e. portions with a marginal tax rate greater than $100 \%$, are ruled out. ${ }^{1}$

The consumers' budget constraint implies $z(y)=c$. Therefore, their optimum effort level is given by:

$$
e=e^{*}(a, b)=\arg \max _{e \in[0,1 / a]} z(y)-b e^{\eta} / \eta
$$

The government tries to maximize an objective function which reflects a concern for income distribution, as well as to provide for a public good. Specifically I assume that the social welfare function is given by

$$
W=\left[\left(\frac{1}{q} \sum_{i=1}^{q} z\left(y_{i}\right)^{\gamma}\right)^{\psi / \gamma}+\lambda\left(\frac{T}{q}\right)^{\psi}\right]^{1 / \psi}
$$

where $T$ is net total tax receipts:

$$
T=\sum_{i=1}^{q} y_{i}-z\left(y_{i}\right) .
$$

The social welfare function is therefore a CES aggregate of total tax receipts per capita, and of an index of average personal disposable income adjusted for inequality in the fashion of Atkinson (1970). ${ }^{2}$ This index is homogeneous of degree one in disposable income, but increases with meanpreserving redistributions. Concern for inequality is captured by $\gamma \leq 1$.

\footnotetext{
${ }^{1}$ There is no a priori problem with having such portions, except that allowing them tends to spuriously increase the number of local optima. This is because marginal tax rates in excess of $100 \%$ may generate configurations where disposable income falls with pre-tax income beyond a certain income level. Nobody would get a pre-tax income greater than this threshold, and marginal changes in the tax code in this zone typically have no effect. This tends to generate a large number of equivalent local optima. To rule out this possibility we impose that marginal tax rates should not exceed $100 \%$.

${ }^{2}$ Note that the (unobservable) disutility of labor is not taken into account in the social welfare function.
} 
The smaller $\gamma$, the greater inequality aversion. $T / q$ enters because net tax receipts are spent on a public good. $\lambda$ and $\psi$ capture the relative weight of the public good in social welfare and the social elasticity of substitution between (inequality adjusted) disposable income and the public good, respectively.

Starting from a given tax code, the government tries to improve it by implementing a succession of small restricted changes to the tax code. It can only move net income by \pm 0.005 at one kink point at a time. Figure 2 gives an example of a feasible tax changes. If no such tax change improves social welfare, then we have a restricted local optimum, which we consider as a potential equilibrium outcome of the economy.

The next section describes in detail the procedure which was followed in order to compute these RLOs.

\section{Procedure}

An economy is randomly generated with $q$ agents. For each agent, $a$ is drawn over $[0,1]$, and $b$ is independently drawn over $[1,2]$, using a uniform distribution in both cases. The policymaker starts from a randomly determined tax code with $n$ brackets, by randomly selecting disposable income at each of the kink points, subject to the constraint that marginal tax rates cannot exceed $100 \%$.

Given a tax code, I compute the effort level of each individual by a grid search over $[0,1 / a]^{3}$ This allows to compute aggregates such as GDP, tax receipts, and social welfare. If the initial, randomly drawn tax code yields negative tax receipts, another one is drawn, until one ends up with a revenuegenerating scheme.

Then a restricted local optimum is reached by the following iteration procedure: a number $m$ is randomly selected, and $z(m / n)$, i.e. disposable income that the kink point between bracket $m$ and bracket $m+1$, is increased by 0.005 , as described in the previous section. Social welfare using this new

\footnotetext{
${ }^{3}$ The step which was used was 0.01 .
} 
tax code is computed and compared to its value under the previous tax code. If an improvement is obtained, then the new tax code is substituted for the old one, and the procedure is repeated. Otherwise, one decreases $z(m / n)$ by 0.005 . If no improvement is obtained, the procedure considers similar changes for other possible kink points, in the following order: $(m+1) / n,(m+$ $2) / n, \ldots, 1,0,1 / n, \ldots,(m-1) / n$. As soon as an improvement is obtained, the new tax code replaces the old one and the procedure is repeated. If no improvement is obtained for all possible kink points, one has reached an RLO and the procedure stops.

For each set of parameter values, I have generated 10 different random economies. For each of them, I have considered all the values of $n$ between $n=1$ and $n=6$. Given an economy and a value of $n$, the search for a local optimum is repeated $15 n$ times, starting from $15 n$ different randomly generated initial tax codes. ${ }^{4}$

Given computational errors, from a strictly numerical perspective the $15 n$ searches are likely to yield $15 n$ different outcomes. One can then in principle look at all these tax codes and consider those which are "close" as the same RLO. However, this is time consuming and not very precise. In order to give a precise content to such an "optical" method, I use the following kernel transformation. For $k=1, \ldots, 15 n$, the corresponding RLO is a vector $X_{k} \in[0,1]^{n+1}$. For any vector $X$ the kernel function is then given by:

$$
K(X)=\sum_{k=1}^{15 n} \frac{1}{\sigma} \exp \left(-\frac{\left\|X-X_{k}\right\|^{2}}{2 \sigma^{2}}\right) .
$$

I then use the local optima of $K(X)$ as my estimates of local optima. ${ }^{5}$

\footnotetext{
${ }^{4}$ In order to preserve the relative density of initial tax codes in the tax code space as $n$ goes up, one would need to have the number of searches to increase like $x^{n}$. However, this is exteremly costly in terms of computer time, so we have chosen a more modest option. Intuitively, it implies that one is more likely to miss some local optima as $n$ goes up, thus leading us to underestimate the effect of $n$ on the number of local optima.

${ }^{5}$ To compute these local optima, we use the actual values of $X_{k}$ as starting point for an iteration procedure using the Newton-Raphson method. This makes sense since the
} 
The precision of this method is captured by $\sigma$. If $\sigma$ is very small, there will be as many humps as values of $X_{k}$. If $\sigma$ is very large, $K($.$) is very$ smooth and has very few humps. After various experimentations I have used $\sigma=0.03 \sqrt{n+1}^{6}$

\section{Results}

Table 1 reports the average number of restricted local optima found by the procedure. Each number is the average over 10 randomly selected economies. The key finding which emerges from Table 1 is that the number of RLOs typically raises sharply with the number of tax brackets $n$, while it is low for a single tax bracket. Therefore, economies with more complex tax systems are more likely to be stuck at a history-dependent, inefficient local RLO. However, this ceases to be true at very high elasticities of labor supply. For $\eta=1.5$, the number of local optima goes up with the number of brackets, but then it falls.

Furthermore, the number of optima first increases and then falls with the elasticity of labor supply $1 /(\eta-1)$. Thus, for $n=3$, for example, the average number of optima increases from 2.4 to 6.2 as $\eta$ falls from 10 to 2 , but it then falls to 4.0 as $\eta$ falls to 1.5 .

How could one explain such nonmonotonicity? When $\eta$ goes down, the effect of a local reform is more complex, because people are more likely to move across brackets. Kauffman's findings suggest that this should increase the number of optima as the objective function becomes more 'rugged' in the policy space. This is indeed what I find except for the lowest value of $\eta$. As $\eta$ falls, an increasing proportion of agents elect a corner solution $(e=0$ or $e=1 / a$ ) for their effort level. This tends to reduce the effective response of

local optima should be 'close' to the computed ones. If two initial values of $X_{k}$ lead to two estimated local optima for $K(X)$ such that the Euclidian distance between them is lower than $\sigma / 3$, then they are considered as the same local optimum.

${ }^{6}$ Proportionality between $\sigma^{2}$ and the dimension of the relevant space guarantees that as $n$ goes up, the average 'tolerance' per kink point is kept constant. 
effort levels to marginal changes in the tax code. In other words, the true elasticity of labor supply does not necessarily fall when $\eta$ becomes small. It falls for agents who are not at a corner solution but on the other hands more agents are at a corner solution. Thus, that the number of optima eventually falls as $\eta$ reaches 1.5 does not necessarily contradict our basic intuition.

\begin{tabular}{lllll}
\hline \hline$n \backslash \eta$ & 10 & 5 & 2 & 1.5 \\
\hline 1 & $1(0.0)$ & $1(0.0)$ & $2.0(0.2)$ & $2.0(0.1)$ \\
2 & $2.2(0.2)$ & $3.7(0.3)$ & $4.1(0.3)$ & $2.7(0.3)$ \\
3 & $2.4(0.4)$ & $4.9(0.4)$ & $6.2(0.3)$ & $4.0(0.4)$ \\
4 & $2.6(0.3)$ & $4.5(0.4)$ & $8.7(0.4)$ & $6.7(0.7)$ \\
5 & $3.0(0.5)$ & $4.2(0.25)$ & $8.7(0.4)$ & $5.5(0.4)$ \\
6 & $3.9(0.4)$ & $5.3(0.4)$ & $10.3(0.7)$ & $4.1(0.5)$ \\
\hline \hline
\end{tabular}

Table 1 - Number of optima as a function of $n$ and $\eta$.

Table 2 reports how the complexity of the tax system affects its average performance. This average performance is defined as the average value, denoted by $W(n)$, of the social welfare function over all $15 n$ searches for RLOs that were made for a given economy. In other words, it is a weighted average of the objective function across local optima, where each optimum is weighted by the frequency with which it was reached. For any economy I then compute the relative performance of the tax system with $n$ brackets as $R(n)=W(n) / W(1)$, the ratio between the average performance at $n$ and the average performance with 1 brackets. Table 2 then reports the average value of $R(n)$ across the 10 randomly generated economies.

The results in Table 2 are striking. For $\eta=2,5$, and 10, performance quickly falls when the number of tax brackets becomes too large. That is, the extra losses from being trapped at new, inefficient optima which did not exist for more simple tax systems outweigh the extra gains from a better fine-tuning of tax policy. Furthermore, this effect is stronger, the lower $\eta$, i.e. the greater the effect of an increase in $n$ on the number of optima. For example, an increase in the number of tax brackets from $n=1$ to $n=6$ reduces average performance by $5.4 \%$ if $\eta=10$, but it reduces it by $12.1 \%$ 
for $\eta=5$ and by $23.2 \%$ for $\eta=2$. Consequently, optimal tax systems have few brackets. The best performing value of $n$ is 3 for $\eta=10$, and 2 for $\eta=2$ or $\eta=5$.

The story is pretty different, however, when labor supply elasticity becomes very large. For $\eta=1.5$, more complex tax systems always perform better than $n=1$. While the optimal number of brackets is $n=3$, the loss from increasing tax complexity beyond the optimum are smaller than in the $\eta=2$ case (some $8 \%$ instead of $23 \%$ ). One can speculate that these results are again due to the fact that an increasing number of agents elect a corner solution for effort, thus being inelastic at the margin.

The results are in sharp contrast to what one would expect in a first best world. In such a world, an increase in the number of tax brackets by an integer multiplicative factor would always increase welfare, since the old tax rates could be replicated using the new tax system. ${ }^{7}$ But this only holds if the optimum optimorum is implemented. If instead equilibrium policy is defined as an RLO reached by a tatonment procedure, then average performance can go down because new inefficient RLOs are introduced when the tax system's complexity is increased.

\begin{tabular}{lllll}
\hline \hline$n \backslash \eta$ & 10 & 5 & 2 & 1.5 \\
\hline 1 & 1.0 & 1.0 & 1.0 & 1.0 \\
2 & $1.008(0.003)$ & $1.001(0.006)$ & $1.009(0.015)$ & $1.08(0.013)$ \\
3 & $1.01(0.003)$ & $0.976(0.006)$ & $0.925(0.015)$ & $1.09(0.019)$ \\
4 & $0.999(0.004)$ & $0.957(0.009)$ & $0.861(0.015)$ & $1.05(0.026)$ \\
5 & $0.972(0.006)$ & $0.913(0.006)$ & $0.81(0.014)$ & $1.04(0.026)$ \\
6 & $0.946(0.006)$ & $0.879(0.01)$ & $0.768(0.019)$ & $1.007(0.033)$ \\
\hline \hline
\end{tabular}

Table 2 - Average relative performance of tax systems.

Table 3 provides an example of the 4 policy outcomes that are found for one of the economies with $n=3$ and $\eta=5$. For each local optimum,

\footnotetext{
${ }^{7}$ Note that in terms of the global optimum $n=3$ does not necessarily dominate $n=2$, since the kink points are pegged at $1 / n$, implying that one cannot typically replicate a tax code with $n=2$ with a 3 -bracket tax code.
} 
Columns 2 to 5 respectively report the value of the objective function, total tax receipts, GDP, and the frequency with which the optimum is reached. Column 6 gives the net income of an agent with zero pre-tax income, i.e. the transfer made to the poorest. Columns 7 to 9 give the marginal tax rate in each bracket, from the bottom to the top of the distribution of income.

The most frequently reached RLO (Equilibrium \#1) is also the most desirable one. It has the property that marginal tax rates fall when income goes up, in accordance with the findings of Mirrlees (1971). In $26.6 \%$ of the cases, however, the economy converges to an equilibrium which is much more redistributive, with a marginal tax rate of $100 \%$ for top earners, and slightly higher marginal tax rates than Equilibrium \#1 for other agents. Tax receipts are only slightly above their level in the other equilibrium, so that the transfer to the poorest is only slightly larger. On the other hand, GDP is $12 \%$ lower, so that on net this equilibrium performs much worse than the other one. Finally, there exists two other rare equilibria. The first one has substantially higher tax receipts than the other two, at the cost of confiscatory tax rates for bottom and low earners. It subsidizes high earnings at the margin, thus inducing a high effort level by many agents, but still extorts high taxes from the wealthy, as the average tax rate is about $60 \%$ for top earners. These high distortions strongly reduce GDP, while there is little redistribution; but tax receipts and thus the level of public goods is higher than in the other two equilibria. Finally, Equilibrium \#4 also virtually expropriates all low and medium income workers, while giving strong work incentives at the top. It has lower tax receipts than other equilibria, but a higher GDP than equilibria \#2 and \#3.

\begin{tabular}{lllllllll}
\hline \hline Eqbm \# & $V$ & $T$ & $Y$ & $F$ & $z(0)$ & MTR1 & MTR2 & MTR3 \\
\hline 1 & .601 & .14 & .352 & .683 & .107 & 0.85 & 0.57 & 0.17 \\
2 & 0.562 & 0.15 & 0.31 & 0.266 & 0.108 & 0.856 & 0.601 & 1.0 \\
3 & 0.39 & 0.197 & 0.26 & 0.033 & 0.025 & 0.948 & 1.0 & -0.13 \\
4 & 0.24 & 0.138 & 0.326 & 0.017 & 0.01 & 0.985 & 0.999 & -1.46 \\
\hline \hline
\end{tabular}

Table 3 - Characteristics of four RLOs in one of the simulated economies, 
with $n=3$ and $\eta=5$.

As table 3 suggests, there exist local optima that perform very poorly, although those in Table 3 tend to be quite infrequent. Table 4 computes the maximum damage of history dependence as measured by the percentage difference between the value of the objective function at the best optimum and its value at the worst one. As Table 4 shows, this loss can be huge and tends to go up with the system's complexity as well as labor supply elasticity, with again an exception at very low elasticities.

\begin{tabular}{lllll}
\hline \hline$n \backslash \eta$ & 10 & 5 & 2 & 1.5 \\
\hline 1 & 0 & 0 & 14.9 & 35.8 \\
2 & 18.8 & 39.7 & 60.4 & 29.1 \\
3 & 19.4 & 58.1 & 74.2 & 39.5 \\
4 & 32 & 76.2 & 80.2 & 50.9 \\
5 & 45.4 & 78.7 & 83.1 & 57.2 \\
6 & 51.5 & 71.1 & 84.7 & 59.5 \\
\hline \hline
\end{tabular}

Table 4- Maximum loss (\%)

As this pattern may just be driven by very infrequent poor outcomes, Table 5 looks at the average loss, i.e. at the difference between the average performance of searches and welfare at the best optimum. It confirms that losses can be quite large and are overall larger, the more complex the system and the greater the elasticity of labor supply.

\begin{tabular}{lllll}
\hline \hline$n \backslash \eta$ & 10 & 5 & 2 & 1.5 \\
\hline 1 & 0 & 0 & 5.9 & 11.2 \\
2 & 1.4 & 4.6 & 9.0 & 7.5 \\
3 & 1.9 & 6.7 & 20.5 & 10.7 \\
4 & 3.2 & 9.5 & 27.0 & 15.7 \\
5 & 6.1 & 13.4 & 31.7 & 18.3 \\
6 & 8.7 & 17.1 & 34.9 & 22.2 \\
\hline \hline
\end{tabular}

Table 5 - Average loss (\%) 


\section{Robustness checks}

In order to check the robustness of my results, I have run a number of simulations with different parameter values. ${ }^{8}$

First, I have allowed for lower inequality aversion by running simulations with $\gamma=-1$ and $\gamma=-0.5$ instead of $\gamma=-2$. The results are quite similar to the ones reported above (see Appendix), with the exception that the number of optima does not systematically rise monotonically with $n$ for $\eta=2,5,10$. In some cases, it slightly falls for $n \geq 4$, although this is often not significant. All the other patterns are confirmed, in particular the "optimal" number of brackets never exceeds 3 .

Second, I have changed the parameter $\psi$ which captures the degree of complementarity between public expenditure and inequality-adjusted GDP in the social welfare function, by trying $\psi=-0.5$ instead of $\psi=0.5$. Results are again similar.

Finally, I have tried with other weights of public expenditure in the social welfare function $(\lambda=2$ and $\lambda=0.5)$. Results are again similar.

\section{Conclusion}

Government policy is based on a large set of interventions: taxes, subsidies, regulations, and discretionary interventions. This paper has explored the idea that they cannot all be changed at the same time, and that the government cannot compute its optimal mix. Rather, it can implement marginal reforms, and keep them if they prove satisfactory.

In the context of tax policy, we have shown that this piece-meal, tatonment approach to economic policy may generate history dependence, in that several local optima may be reached, and which one is reached depends on initial conditions. History dependence is stronger (i.e. there are more local

\footnotetext{
${ }^{8}$ In these checks, 4 economies instead of 10 were used for each parameter set. Results are reported in the Appendix.
} 
optima), the more complex the design of economic policy, and the greater the interaction of policy instruments with one another-which in my tax model means that economic agents have a more elastic behavior.

Furthermore, while a more complex policy design may in principle improve outcomes because it increases the value of the best optimum, in practice a complex system may have a lower performance. This is because complexity increases the number of local optima, so the search process may more often yield unsatisfactory outcomes, implying that welfare is lower on average. Indeed, in the context of my tax model, the best performing number of tax brackets is typically equal to 2 or 3 .

In the future, I plan to apply similar analysis to other aspects of economic policy, such as environmental regulation or active labor market policies, for example. 


\section{REFERENCES}

Atkinson, Anthony B. (1970) "On the measurement of inequality", Journal of Economic Theory, 2(3), 244-63

Brock, William and Steven Durlauf (2001), "Discrete choice with local interactions", Review of Economic Studies, 68(2), 235-60

Durlauf, Steven (1993), "Nonergodic economic growth", Review of Economic Studies 60(2), 349-66

Kauffman, Stuart (1985), The Origins of Order, Oxford U. Press

Mirrlees, James (1971), "An exploration in the theory of optimal taxation", Review of Economic Studies, 38(114), 175-208 
APPENDIX - Robustness checks

\begin{tabular}{lllll}
\hline \hline$n \backslash \eta$ & 10 & 5 & 2 & 1.5 \\
\hline 1 & $1(0.0)$ & $1(0.0)$ & $1.25(0.25)$ & $2(0)$ \\
2 & $2(0.0)$ & $3.75(0.5)$ & $4.25(0.25)$ & $3.0(0.4)$ \\
3 & $2.75(0.5)$ & $6.0(0.7)$ & $7(0.7)$ & $5.25(0.5)$ \\
4 & $5.25(0.75)$ & $6.5(0.6)$ & $9.25(0.5)$ & $6.25(0.9)$ \\
5 & $4.5(0.3)$ & $5.25(0.6)$ & $8.75(1.3)$ & $5.5(1.0)$ \\
6 & $4.0(0.7)$ & $5(0.0)$ & $7.75(0.5)$ & $4(0.7)$ \\
\hline
\end{tabular}

$\overline{\text { Table A1 - Number of optima as a function of } n \text { and } \eta} ; \gamma=-1$

\begin{tabular}{lllll}
\hline \hline$n \backslash \eta$ & 10 & 5 & 2 & 1.5 \\
\hline 1 & 1.0 & 1.0 & 1.0 & 1.0 \\
2 & 1.003 & 0.99 & 0.96 & 1.06 \\
3 & 1.009 & 0.98 & 0.84 & 0.99 \\
4 & 0.99 & 0.92 & 0.79 & 0.95 \\
5 & 0.97 & 0.9 & 0.7 & 0.87 \\
6 & 0.95 & 0.85 & 0.69 & 0.83 \\
\hline \hline
\end{tabular}

Table A2 - Average relative performance of tax systems; $\gamma=-1$

\begin{tabular}{lllll}
\hline \hline$n \backslash \eta$ & 10 & 5 & 2 & 1.5 \\
\hline 1 & $1(0.0)$ & $1(0.0)$ & $2(0.0)$ & $2.5(0.3)$ \\
2 & $1.5(0.3)$ & $3.5(0.3)$ & $4.0(0.4)$ & $3.25(0.5)$ \\
3 & $3.5(0.9)$ & $4.0(0.7)$ & $7.25(0.6)$ & $5.75(0.6)$ \\
4 & $4.5(0.6)$ & $5.25(0.9)$ & $9.5(0.3)$ & $7.75(1.0)$ \\
5 & $5.0(0.7)$ & $6.25(1.25)$ & $10(0.9)$ & $5.25(1.1)$ \\
6 & $4.25(0.6)$ & $5.25(0.6)$ & $10.25(1.6)$ & $6.75(0.8)$ \\
\hline
\end{tabular}

$\overline{\text { Table A3- Number of optima as a function of } n \text { and } \eta} ; \gamma=-0.5$

\begin{tabular}{lllll}
\hline \hline$n \backslash \eta$ & 10 & 5 & 2 & 1.5 \\
\hline 1 & 1.0 & 1.0 & 1.0 & 1.0 \\
2 & 1.01 & 0.99 & 0.99 & 1.08 \\
3 & 0.999 & 0.96 & 0.85 & 1.09 \\
4 & 0.987 & 0.92 & 0.79 & 1.016 \\
5 & 0.973 & 0.86 & 0.74 & 0.99 \\
6 & 0.96 & 0.86 & 0.7 & 0.94 \\
\hline \hline
\end{tabular}

Table A4 - Average relative performance of tax systems; $\gamma=-0.5$ 


\begin{tabular}{lllll}
\hline \hline$n \backslash \eta$ & 10 & 5 & 2 & 1.5 \\
\hline 1 & $1(0.0)$ & $1.25(0.25)$ & $1.5(0.3)$ & $2.75(0.25)$ \\
2 & $2.5(0.5)$ & $3.5(0.6)$ & $3.5(0.3)$ & $2.75(0.25)$ \\
3 & $2.75(0.5)$ & $3(1.1)$ & $7(0.6)$ & $5(0.7)$ \\
4 & $3.25(0.5)$ & $5.5(0.6)$ & $9.25(0.6)$ & $6.25(0.8)$ \\
5 & $2.75(0.25)$ & $5.25(0.9)$ & $8.5(1.2)$ & $5.25(0.8)$ \\
6 & $4.25(1.2)$ & $5.5(0.9)$ & $9(1.3)$ & $5.75(1.3)$ \\
\hline
\end{tabular}

$\overline{\text { Table A5 - Number of optima as a function of } n \text { and } \eta ;} \psi=-0.5$

\begin{tabular}{lllll}
\hline \hline$n \backslash \eta$ & 10 & 5 & 2 & 1.5 \\
\hline 1 & 1 & 1 & 1 & 1 \\
2 & 1.002 & 1.017 & 0.95 & 1.22 \\
3 & 1.007 & 1.007 & 0.89 & 1.23 \\
4 & 0.983 & 0.93 & 0.84 & 1.155 \\
5 & 0.971 & 0.88 & 0.79 & 1.18 \\
6 & 0.944 & 0.857 & 0.73 & 1.14 \\
\hline \hline
\end{tabular}

Table A6 - Average relative performance of tax systems; $\psi=-0.5$

\begin{tabular}{lllll}
\hline \hline$n \backslash \eta$ & 10 & 5 & 2 & 1.5 \\
\hline 1 & $1(0.0)$ & $1(0.0)$ & $1.5(0.3)$ & $2(0.0)$ \\
2 & $1.5(0.3)$ & $3.25(0.5)$ & $4(0.4)$ & $2.75(0.25)$ \\
3 & $1.75(0.5)$ & $3.0(0.7)$ & $6.75(0.5)$ & $5.5(0.95)$ \\
4 & $2.0(0.4)$ & $3.0(0.7)$ & $8.5(0.9)$ & $7.25(0.75)$ \\
5 & $2.75(0.5)$ & $3(0.0)$ & $8.0(0.7)$ & $6.25(0.5)$ \\
6 & $3.0(0.4)$ & $5.0(0.4)$ & $8.25(1.8)$ & $6.75(1.0)$ \\
\hline \hline
\end{tabular}

Table A7 - Number of optima as a function of $n$ and $\eta ; \lambda=2$

\begin{tabular}{lllll}
\hline \hline$n \backslash \eta$ & 10 & 5 & 2 & 1.5 \\
\hline 1 & 1 & 1 & 1 & 1 \\
2 & 1.01 & 1.023 & 0.995 & 1.135 \\
3 & 1.015 & 1.01 & 0.936 & 1.071 \\
4 & 0.999 & 0.998 & 0.847 & 1.037 \\
5 & 0.999 & 0.987 & 0.806 & 1.011 \\
6 & 0.995 & 0.955 & 0.824 & 1.014 \\
\hline \hline
\end{tabular}

Table A8 - Average relative performance of tax systems; $\lambda=2$ 


\begin{tabular}{lllll}
\hline \hline$n \backslash \eta$ & 10 & 5 & 2 & 1.5 \\
\hline 1 & $1(0.0)$ & $1(0.0)$ & $1.75(0.25)$ & $2.0(0.41)$ \\
2 & $1.25(0.25)$ & $3.75(0.48)$ & $4(0.0)$ & $2.75(0.25)$ \\
3 & $3.0(0.41)$ & $6.5(0.29)$ & $7.5(0.96)$ & $6.0(0.58)$ \\
4 & $5.5(0.29)$ & $7.25(0.63)$ & $9.0(0.82)$ & $8.25(1.65)$ \\
5 & $7.0(0.41)$ & $5.75(0.25)$ & $10.5(2.18)$ & $6.25(1.18)$ \\
6 & $5.25(0.75)$ & $7.5(0.87)$ & $9.0(0.71)$ & $7.25(1.44)$ \\
\hline Table & A9 - Number of optima as a function of $n$ and $\eta ; \lambda$
\end{tabular}$=0.5$

\begin{tabular}{lllll}
\hline \hline$n \backslash \eta$ & 10 & 5 & 2 & 1.5 \\
\hline 1 & 1 & 1 & 1 & 1 \\
2 & 1.016 & 0.969 & 0.925 & 1.06 \\
3 & 0.998 & 0.958 & 0.836 & 1.02 \\
4 & 0.963 & 0.879 & 0.733 & 0.956 \\
5 & 0.935 & 0.851 & 0.693 & 0.915 \\
6 & 0.865 & 0.794 & 0.659 & 0.899 \\
\hline \hline
\end{tabular}

Table A10 - Average relative performance of tax systems; $\lambda=0.5$ 
Net income

Figure 1

Gross income 
Net income

Figure 2:

Example of a feasible reform

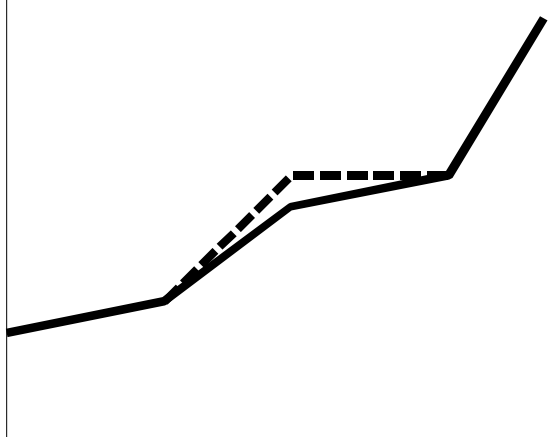

Gross income 